TRANSACTIONS OF THE

AMERICAN MATHEMATICAL SOCIETY

Volume 357, Number 8, Pages 3359-3373

S 0002-9947(05)03657-3

Article electronically published on March 10, 2005

\title{
ON COMPACT SYMPLECTIC MANIFOLDS WITH LIE GROUP SYMMETRIES
}

\author{
DANIEL GUAN
}

\begin{abstract}
In this note we give a structure theorem for a finite-dimensional subgroup of the automorphism group of a compact symplectic manifold. An application of this result is a simpler and more transparent proof of the classification of compact homogeneous spaces with invariant symplectic structures. We also give another proof of the classification from the general theory of compact homogeneous spaces which leads us to a splitting conjecture on compact homogeneous spaces with symplectic structures (which are not necessary invariant under the group action) that makes the classification of this kind of manifold possible.
\end{abstract}

\section{INTRODUCTION}

A symplectic manifold is a smooth $2 n$-dimensional manifold $M$ equipped with a closed 2-form which is nondegenerate everywhere. Recently there has been much progress in the area of symplectic manifolds and group actions. I am concerned with the classical problem of classifying compact homogeneous spaces with symplectic structures.

If a symplectic structure is invariant under an action of a transitive Lie group, one difficulty is that nothing is known about the transitive group and the isotropy group (cf. [3, 4], 19]). In the Kähler case we know that the isometric group is compact. In this paper we prove following theorem:

Theorem. Every finite-dimensional Lie subgroup of the automorphism group of a compact symplectic manifold is locally a product of a compact semisimple group and a 2-step solvable group $R$. Moreover, the adjoint representation of $R$ on $R^{\prime}$ is a subgroup of a compact torus.

An application of this result is a simpler, more transparent and also more prospective proof of the classification of the compact homogeneous manifolds with invariant symplectic structures.

I am also interested in the structure of compact homogeneous manifolds with symplectic structures that are not necessarily invariant under the group action.

Received by the editors May 22, 2002 and, in revised form, February 26, 2004.

2000 Mathematics Subject Classification. Primary 53C15, 57S25, 53C30, 22E99, 15A75.

Key words and phrases. Invariant structure, homogeneous space, product, fiber bundles, symplectic manifolds, splittings, prealgebraic group, decompositions, modification, Lie group, symplectic algebra, compact manifolds, uniform discrete subgroups, classifications, locally flat parallelizable manifolds.

This work was supported by NSF Grant DMS-9627434 and DMS-0103282. 
In [12], [13] we proved the following theorem:

Proposition 1. Every compact homogeneous complex manifold with a 2-cohomology class $\omega$ such that $\omega^{n}$ is not zero in the top cohomology is a product of a rational homogeneous space and a complex parallelizable solv-manifold with a right invariant symplectic structure on its universal covering.

This generalizes the result of 2] for the Kähler case (one does not assume that the Kähler form is invariant).

These results suggest further study in two directions along the lines of Proposition 1. One is the classification of compact complex homogeneous space; the other is the classification of compact homogeneous space with a symplectic structure. The first problem can be solved by the method used in [14, 18]. In 14 we proved that every compact complex homogeneous space with an invariant volume is a torus bundle over a product of a rational homogeneous space and a complex parallelizable manifold, and in [18] we classified compact complex homogeneous spaces of 1-step. In the present paper we are also working in the direction of the second problem. This is quite analogous to the results in [12, 13]. In the case of the existence of a symplectic structure which is invariant under the action of a transitive Lie group, one has from [24:

Proposition 2. Every compact homogeneous space with an invariant symplectic structure is a product of a rational homogeneous space and a torus with invariant symplectic structures.

In this classification, the tori which occur are not necessarily standard. Not knowing about [24] we reproved this same result in [16], 17]. The following conjecture arises naturally from our arguments for the proof of the above proposition:

Conjecture. If $G / H$ is a compact homogeneous space with a symplectic structure, then $G / H$ is diffeomorphic to a product of a rational homogeneous space and a finite quotient of a compact locally flat parallelizable manifold with a symplectic structure.

We call a manifold $N$ locally flat parallelizable if $N=G / H$ for a simply connected Lie group $G$ which is diffeomorphic to $\mathbf{R}^{k}$ for some integer $k$ and $H$ is a uniform discrete subgroup.

In our future work we will attempt to prove this conjecture and to classify the compact locally flat parallelizable manifolds with a symplectic structure.

When I wrote [16] and 17] in 1997 I was unaware of Zwart and Boothby's paper 24. Our proof follows the line of the section 3-we proved the diffeomorphism in [16], then the symplectic isomorphism in [17. Motivated by our methods in [16] we obtained our Theorem in 1997, which also gave a much simpler proof of Proposition 2. Altogether we have three proofs.

I recently became aware of [24] and although it includes the results in [16] and [17], the proofs are completely different. I still think that it is worthy of publishing our original proof of the above proposition so as to provide the motivation for our Conjecture and to show the relationship to the general compact homogeneous manifold theory.

Our original proof of Proposition 2 is based on the recent results of Gorbatsevich [7], [8], [9], [10] which should lead to more applications to the classifications 
of homogeneous spaces. Combining Gorbatsevich's results with moment map arguments (see [19]) in section 3 we first obtained the splitting of the double normalizer fibration [7] and then we applied Gorbatsevich's modification [10] and an earlier version of our Theorem to obtain the differential structure of the solvable compact symplectic manifolds. The symplectic structure could be obtained by completely integrable systems which we omit here. Therefore, our proof is more geometric. The proof of 24 is more algebraic, since they studied the group fixing the given 2-form in the adjoint action $H$ in 24 and applied the representation theory of unipotent groups.

One might ask whether there is a compact semisimple Lie group $S$ in the automorphism group such that all the compact semisimple factors occured in our Theorem are conjugate to subgroups of $S$. If the answer is yes, then we have a new symplectic invariant. This would be a generalization of a similar result from the Kähler case. One might also ask whether the maximal compact semisimple Lie groups $S$ above come from those in the holomorphic automorphism group if the manifolds are Kähler.

Another consequence of our Theorem is that we can start to consider the classification of compact almost homogeneous symplectic manifolds and compact symplectic manifolds with hypersurfaces orbits.

\section{The PROOF OF OUR THEOREM}

2.1. Preliminaries. Let $(M, \omega)$ be a symplectic manifold and let $G$ be a Lie group of symplectic diffeomorphisms of $M$, i.e., a smooth action $G \times M \rightarrow M$ such that $g^{*} \omega=\omega$ for all $g \in G$. Let $\operatorname{Ham}_{l o c}(M)$ be the set of smooth vector fields $X$ on $M$ such that $L_{X} \omega=0$. We have the following sequence:

$$
0 \rightarrow \mathbf{R} \stackrel{i}{\rightarrow} C^{\infty}(M) \stackrel{\text { sgrad }}{\rightarrow} \operatorname{Ham}_{l o c}(M) \stackrel{\alpha}{\leftarrow} \mathcal{G},
$$

where $i$ realizes the real numbers as constant functions, the skew-gradient $\operatorname{sgrad}(f)$ is a vector field $X_{f}$ such that

$$
i_{X_{f}} \omega=\omega\left(X_{f},\right)=d f
$$

and $\alpha$ is the natural Lie algebra homomorphism arising from the $G$-action. The associated Lie algebra structure $\{$,$\} on C^{\infty}(M)$ is defined by

$$
\{f, g\}=\omega(\operatorname{sgrad}(f), \operatorname{sgrad}(g)) .
$$

It follows that sgrad: $C^{\infty}(M) \rightarrow \operatorname{Ham}_{l o c}(M)$ is a Lie algebra homomorphism, and we are confronted with a lifting question: Does there exist a Lie morphism $\lambda: \mathcal{G} \rightarrow C^{\infty}(M)$ such that sgrado $\lambda=\alpha$ ? If such a lifting exists, we refer to the $G$-action as a Poisson action (with regard to the lifting). In this case the $G$-equivariant dual map

$$
\Phi: M \rightarrow \mathcal{G}^{*}, \quad \Phi(x)(\xi)=\lambda(\xi)(x)
$$

is called the moment map. If every $\mathcal{G}$-field is the skew-gradient of some function, i.e., if for every $\xi \in \mathcal{G}$ the associated vector field is of the form $\xi_{M}=\operatorname{sgrad}\left(f_{\xi}\right)$, then the $G$-action is called a Hamiltonian action (our definition is different from the one in [11]). 
The following is a list of elementary observations in the above setting (see [11):

(1) Let $G^{\prime}$ be the commutator of $G$. Then the $G^{\prime}$-action is Hamiltonian, and if $G$ is a semisimple group, then it induces a Poisson action (see also [11 p. 185]).

(2) Suppose that $\xi \in \mathcal{G}$ can be lifted. Then

$$
\left\{x \in M \mid d f_{\xi}(x)=0\right\}=\left\{x \in M \mid \xi_{M}(x)=0\right\} .
$$

(3) If the $G$-action is Poisson with moment map $\Phi: M \rightarrow \mathcal{G}^{*}$, then

$$
\operatorname{ker}\left(d \Phi_{x}\right)=\left\{v \in T_{x} M \mid \omega_{x}(v, w)=0 \text { for all } w \in T_{x} G(x)\right\}=\left(T_{x} G(x)\right)^{\perp},
$$

where $\left(T_{x} G(x)\right)^{\perp}$ is the skew-orthogonal complement to the tangent space of the $G$-orbit $G(x)$.

(1), (2) and (3) come from the properties of the Poisson bracket. If $M$ is compact, we might use $C_{0}^{\infty}(M)=\left\{\left.f \in C^{\infty}(M)\right|_{\int_{M} f \omega^{n}=0}\right\}$ instead of $C^{\infty}(M)$ in (1), and we see that $G^{\prime}$ is actually Poisson by the argument of [11, pp. 186-187]. We have:

Lemma 1. If $M$ is compact, then the Lie algebra of the exact Hamiltonian group of $M$ is $C_{0}^{\infty}$. The group is "compact" in the sense that its Lie algebra has an invariant positive definite inner product $(f, g)=\int_{M} f g \omega^{n}$.

Proof. It is known that the exact Hamiltonian group of $M$ is generated by functions $h$ with $\int_{M} h \omega^{n}=0$. All these kind of functions consist of an infinite-dimensional Lie algebra since

$$
\int_{M}\{f, g\} \omega^{n}=\int_{M} d f \wedge d g \wedge \omega^{n-1}=0 .
$$

This metric is invariant since

$$
\begin{aligned}
& (\{h, f\}, g)+(f,\{h, g\}) \\
& =\int_{M}(g d h \wedge d f+f d h \wedge d g) \wedge \omega^{n-1} \\
& =\int_{M} d(f g) \wedge d h \wedge \omega^{n-1}=0 .
\end{aligned}
$$

For a further application of this section, we also list the following property:

(4) If $G$ is as in (3) and $G(x)=G / H$ is a generic orbit with moment fibering

$$
\left.\Phi\right|_{G(x)}: G / H \rightarrow G / J=G(\Phi(x)), \quad x \in M,
$$

then $H^{0} \triangleleft J^{0}$ and $J^{0} / H^{0}$ is abelian (see [11, p. 190]).

2.2. The proof of our Theorem. Now we are ready to prove our Theorem:

Since $G^{\prime}$ is a subgroup of the exact Hamiltonian group, there is an invariant inner product $(f, g)$ on its Lie algebra. Moreover, $G$ acts on the Lie algebra of $G^{\prime}$ and keeps the inner product invariant. The adjoint group of $G$ on $G^{\prime}$ is a subgroup of a compact group and it splits locally into a product of its compact semisimple part and the abelian part. So $G$ is locally a product of a compact semisimple group and a solvable 2 -step Lie group $R$ and the adjoint action of $R$ on $R^{\prime}$ is a subgroup of a torus. 


\section{ON THE CLASSIFICATION OF COMPACT HOMOGENEOUS MANIFOLDS WITH INVARIANT SYMPLECTIC STRUCTURES}

\subsection{Preliminaries.}

3.1.1. Some special manifolds. A rational homogeneous manifold $Q$ is a compact complex manifold which can be realized as a closed orbit of a linear algebraic group in some projective space. Equivalently, $Q=S / P$, where $S$ is a complex semisimple Lie group and $P$ a parabolic subgroup, i.e., a subgroup of $S$ which contains a maximal connected solvable subgroup (Borel group). Every homogeneous rational manifold is simply-connected and is therefore an orbit of a compact group. In general, a quotient $K / L$ with $K$ compact and semisimple carries a $K$-invariant complex structure which is projective algebraic if and only if $L$ is the centralizer $C(T)$ of a torus $T \subset K$.

A parallelizable manifold is the quotient of a Lie group by a discrete subgroup.

A solv-manifold is a homogeneous space with a solvable Lie group.

In our research we are interested in locally flat parallelizable manifolds in the sense that the Lie group is locally flat, i.e., its universal covering is diffeomorphism to $\mathbf{R}^{k}$ for some integer $k$. By the classification of the Lie group, we see that a Lie group $G$ is locally flat if and only if its semisimple part is locally isomorphic to a product of $S L(2, \mathbf{R})$ 's.

3.1.2. Simply connected Lie groups. In this subsection we recall some basic results about simply connected Lie groups (see [22]). First, any simply connected Lie group has a Levi decomposition $G=S R$ with $S$ a semisimple subgroup, $R$ a simply connected maximal solvable normal subgroup and $S \cap R=\{e\}$.

A connected closed subgroup $U \subset G$ is said to be a $k$-subgroup if the manifold $G / U$ is compact. Let $\mathcal{G}$ be the Lie algebra of $G$, and let Int $\mathcal{G}$ be the Lie group of its inner automorphisms. A subalgebra $\mathcal{U} \subset \mathcal{G}$ is said to be compact in $\mathcal{G}$ if the connected subgroup in $\operatorname{Int} \mathcal{G}$ corresponding to the subalgebra $\operatorname{Ad}_{\mathcal{G}} \mathcal{U} \subset \operatorname{Ad} \mathcal{G}$ is compact. A subalgebra $\mathcal{H} \subset \mathcal{G}$ is said to be a $k$-subalgebra if there exists a subalgebra $\mathcal{U}$ which is compact in $\mathcal{G}$, such that $\mathcal{G}=\mathcal{U}+\mathcal{H}$.

Let $\mathcal{G}$ be a semisimple Lie algebra, let $\mathcal{U}$ be a maximal compact subalgebra in $\mathcal{G}$, and let $\mathcal{G}=\mathcal{U}+\mathcal{P}$ be the Cartan decomposition. Let $\theta$ denote an involutive automorphism of $\mathcal{G}$ which is given by the formula $\theta(x+y)=x-y$, where $x \in$ $\mathcal{U}, y \in \mathcal{P}$. Let $\mathcal{H}_{-}$be a maximal abelian subalgebra in $\mathcal{P}$ and let $\mathcal{H}=\mathcal{H}_{+}+\mathcal{H}_{-}$ be a Cartan subalgebra in $\mathcal{G}$ which contains $\mathcal{H}_{-}$and $\mathcal{H}_{+} \subset \mathcal{U}$. We consider the complexification $\mathcal{G}^{\mathbf{C}}$ of $\mathcal{G}$ and let us denote by $\sigma$ the corresponding conjugation in $\mathcal{G}^{\mathbf{C}}$. Then $\mathcal{H}^{\mathbf{C}}$ is a Cartan subalgebra in $\mathcal{G}^{\mathbf{C}}$. Let us denote by $\Sigma \subset\left(\mathcal{H}^{\mathbf{C}}\right)^{*}$ the corresponding system of roots of $\mathcal{G}^{\mathbf{C}}$. If $\alpha \in \Sigma$, then by $\mathcal{G}_{\alpha}$ we denote its root subspace in $\mathcal{G}^{\mathbf{C}}$. The roots of $\Sigma$ are real in the subspace $\mathcal{H}_{*}=i \mathcal{H}_{+}+\mathcal{H}_{-}$. Let us determine on $\left(\mathcal{H}^{\mathbf{C}}\right)^{*}$ an anti-involution $\sigma^{*}$ by the formula

$$
\left(\sigma^{*} \phi\right)(x)=\overline{\phi(\sigma x)} \quad\left(x \in \mathcal{H}^{\mathbf{C}}\right)
$$

If $\alpha \in \Sigma$, then $\sigma^{*} \alpha$ is also a root, and $\sigma \mathcal{G}_{\alpha}=\mathcal{G}_{\sigma^{*} \alpha}$. Let $\Sigma_{0}=\left\{\alpha \in \Sigma, \sigma^{*} \alpha=-\alpha\right\}$.

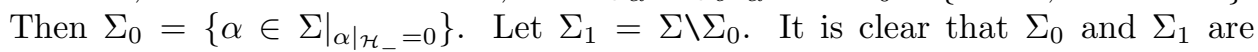
invariant relative to $\sigma^{*}$.

If in $\left(\mathcal{H}^{\mathbf{C}}\right)^{*}$ we introduce an ordering and if $\Delta \subset \Sigma$, let us denote by $\Delta^{+}$and $\Delta^{-}$ the sets of positive and negative roots respectively, which belong to $\Delta$. It is known 
that this ordering may be introduced in such a manner that $\Sigma_{1}^{+}$will be invariant relative to $\sigma^{*}$. Then $\Sigma_{1}^{-}$is also invariant, and $\sigma^{*} \Sigma_{0}^{+}=\Sigma_{0}^{-}$.

The subsystem $\Delta \subset \Sigma$ is said to be closed if for any $\alpha, \beta \in \Delta$ such that $\alpha+\beta \in \Sigma$, we have $\alpha+\beta \in \Delta$. Clearly, $\Sigma_{0}, \Sigma_{1}^{+}$and $\Sigma_{1}^{-}$are closed subsystems.

Set $\mathcal{N}^{\mathbf{C}}=\Sigma_{\alpha \in \Sigma_{1}^{+}} \mathcal{G}_{\alpha}$. Since system $\Sigma_{1}^{+}$is closed, $\mathcal{N}^{\mathbf{C}}$ is a nilpotent subalgebra in $\mathcal{G}^{\mathrm{C}}$. Moreover, $\sigma\left(\mathcal{N}^{\mathrm{C}}\right)=\mathcal{N}^{\mathrm{C}}$. Hence, if we let $\mathcal{N}=\mathcal{N}^{\mathrm{C}} \cap \mathcal{G}, \mathcal{N}^{\mathrm{C}}$ is a complex envelope of $\mathcal{N}$. A so-called Iwasawa decomposition holds; namely:

$$
\mathcal{G}=\mathcal{U}+\mathcal{H}_{-}+\mathcal{N}
$$

or $G=U H_{-} N$ with $H_{-} N$ a maximal triangular subgroup in $G$. Let $\Pi \subset \Sigma^{+}$be a system of simple roots, $\Pi_{0}=\Pi \cap \Sigma_{0}, \Pi_{1}=\Pi \cap \Sigma_{1}$. Then for every $\alpha \in \Pi_{1}$ there exists a $\beta \in \Pi_{1}$ such that $\sigma^{*} \alpha-\beta=\sum_{\gamma \in \Pi_{0}} k_{\gamma} \gamma$, where $k_{\gamma} \geq 0$. If we set $\beta=\tilde{\sigma} \alpha$, then we have an involution $\tilde{\sigma}$ of system $\Pi_{1}$.

An algebra $\mathcal{G}$ is said to be normal if $\mathcal{H}_{-}=\mathcal{H}$. In this case $\Sigma_{1}=\Sigma$ and $\tilde{\sigma}=1$. It is clear that in every complex semisimple Lie algebra there exists exactly one (up to conjugacy) normal real form.

We find it convenient to write a real semisimple Lie algebra $\mathcal{G}$ by means of its Satake scheme. This scheme is constructed as follows. We take the Dynkin diagram of $\mathcal{G}^{\mathbf{C}}$; that is, the diagram of the system $\Pi$. The circles which correspond to elements which belong to $\Pi_{0}$ are blackened; those belonging to $\Pi_{1}$ are left white. In addition, in $\Pi_{1}$ by means of arrows we show pairs of roots which are permuted by the involution $\tilde{\sigma}$.

We call a subgroup $L$ of $G$ a t-subgroup if $H_{-} N N_{R} \subset L$, where $H_{-} N$ is a maximal triangular subgroup of the semisimple part of $G$ and $N_{R}$ is the maximal nilpotent normal subgroup in $R$. To describe a t-subgroup it is sufficient to describe its intersection with the semisimple part of $G$.

Using above terminology, let us describe the well-known method for the construction of t-subalgebras in a semisimple Lie algebra $\mathcal{G}$. Let $\Gamma \subset \Pi_{1}$ be some subset which is invariant relative to $\tilde{\sigma}$. Let us denote by $\Sigma^{\prime}$ the system of roots which can be linearly expressed by the system $\Pi_{0} \cup \Gamma$ and by $\Sigma^{\prime \prime}$ a system of all roots which cannot be expressed linearly by $\Pi_{0} \cup \Gamma$. It is clear that $\Sigma^{\prime}$ and $\Sigma^{\prime \prime}$ are closed systems. In what follows, it is convenient to study an element $x_{\Gamma} \in \mathcal{H}$ which is defined by the formulas

$$
\alpha\left(x_{\Gamma}\right)=\left\{\begin{array}{lll}
0, & \text { if } & \alpha \in \Pi_{0} \cup \Gamma, \\
1, & \text { if } \quad \alpha \in \Pi_{1} \backslash \Gamma .
\end{array}\right.
$$

Clearly, such an element exists and is uniquely determined. We will show that $x_{\Gamma} \in \mathcal{H}_{-}$. In order to do this, one must verify that $\sigma x_{\Gamma}=x_{\Gamma}$ or that $\left(\sigma^{*} \alpha\right)\left(x_{\Gamma}\right)=$ $\overline{\alpha\left(x_{\Gamma}\right)}=\alpha\left(x_{\Gamma}\right)$ for all $\alpha \in \Pi$. For $\alpha \in \Pi_{0}$ this is true because $\sigma^{*} \alpha=-\alpha$. If $\alpha \in \Gamma$, then $\sigma^{*} \alpha=\tilde{\sigma} \alpha+\sum_{\gamma \in \Pi_{0}} k_{\gamma} \gamma$, where $\tilde{\sigma} \alpha \in \Gamma$. For this reason $\left(\sigma^{*} \alpha\right)\left(x_{\Gamma}\right)=$ $(\tilde{\sigma} \alpha)\left(x_{\Gamma}\right)=0=\alpha\left(x_{\Gamma}\right)$. Similarly we verify it for $\alpha \in \Pi_{1} \backslash \Gamma$.

We notice that $\Sigma^{\prime}=\left\{\alpha \in \Sigma, \alpha\left(x_{\Gamma}\right)=0\right\}, \Sigma^{\prime \prime+}=\left\{\alpha \in \Sigma, \alpha\left(x_{\Gamma}\right)>0\right\}$. Because $x_{\Gamma} \in \mathcal{H}_{-}$, it follows at once from what we have proved that $\Sigma^{\prime}$ and $\Sigma^{\prime \prime}{ }^{+}$are invariant relative to $\sigma^{*}$.

Let us denote the centralizer of the element $x_{\Gamma}$ in $\mathcal{G}$ by $\mathcal{Z}_{\Gamma}$. It is clear that $\mathcal{Z}_{\Gamma}^{\mathrm{C}}$ is the centralizer of $x_{\Gamma}$ in $\mathcal{G}^{\mathbf{C}}$ and

$$
\mathcal{Z}_{\Gamma}^{\mathbf{C}}=\mathcal{H}^{\mathbf{C}}+\sum_{\alpha \in \Sigma^{\prime}} \mathcal{G}_{\alpha} .
$$


Let $\mathcal{C}_{\Gamma}$ denote the subspace in $\mathcal{H}$ which is annihilated by all roots in $\Pi_{0} \cup \Gamma$ or, what comes to the same thing, in $\Sigma^{\prime}$, it is the center of $\mathcal{Z}_{\Gamma}$. Also, $\mathcal{Z}_{\Gamma}=\mathcal{C}_{\Gamma}+\mathcal{S}_{\Gamma}$, where $\mathcal{S}_{\Gamma}$ is the semisimple part of $\mathcal{Z}_{\Gamma}$.

Set $\mathcal{N}_{\Gamma}^{\mathrm{C}}=\sum_{\gamma \in \Sigma^{\prime \prime}+} \mathcal{G}_{\gamma}$. It is a nilpotent subalgebra of $\mathcal{G}^{\mathrm{C}}$ which is invariant under $\sigma$. Consequently $\mathcal{N}_{\Gamma}^{\mathrm{C}}$ is a complex envelope of $\mathcal{N}_{\Gamma}=\mathcal{N}_{\Gamma}^{\mathrm{C}} \cap \mathcal{G}$. In particular, $\mathcal{N}_{\emptyset}=\mathcal{N}$.

Finally, set $\mathcal{U}_{\Gamma}=\mathcal{Z}_{\Gamma}+\mathcal{N}_{\Gamma}$. Then $\mathcal{U}_{\Gamma}^{\mathrm{C}}$ is a parabolic subalgebra in $\mathcal{G}$ and $\mathcal{N}_{\Gamma}$ is the nilradical of $\mathcal{U}_{\Gamma}$. Let $\mathcal{C}_{\Gamma}^{+}=\mathcal{C}_{\Gamma} \cap \mathcal{U}, \mathcal{C}_{\Gamma}^{-}=\mathcal{P} \cap \mathcal{C}_{\Gamma}$ and $\mathcal{M}_{\Gamma}=\mathcal{C}_{\Gamma}^{+}+\mathcal{E}_{\Gamma}$ be the maximal compact ideal of $\mathcal{Z}_{\Gamma}$ with $\mathcal{E}_{\Gamma}$ semisimple, and let $\mathcal{Z}_{\Gamma}^{\prime}$ be its complement in $\mathcal{Z}_{\Gamma}$. Then we call a subalgebra

$$
\mathcal{T}=\mathcal{M}+\mathcal{Z}_{\Gamma}^{\prime}+\mathcal{N}_{\Gamma}
$$

with $\mathcal{M}$ a subalgebra of $\mathcal{M}_{\Gamma}$ a standard t-subalgebra. Thus:

Proposition 3. Every t-subalgebra of a semisimple Lie algebra $\mathcal{G}$ is a standard $t$ subalgebra. Moreover, the normalizer of $\mathcal{T}$ in $\mathcal{G}$ is $P(\mathcal{M})+\mathcal{Z}_{\Gamma}^{\prime}+\mathcal{N}_{\Gamma}$, where $P(\mathcal{M})$ is the normalizer of $\mathcal{M}$ in $\mathcal{M}_{\Gamma}$.

3.1.3. Generalization of Tits fibrations. In this subsection we recall some basic results on a generalization of the Tits fibration to compact homogeneous spaces, introduced by V. V. Gorbatsevich [7] which coincides with a fibration considered by Tits [23] in the case of compact complex homogeneous spaces. We call it the double normalizer fibration as in [7] or the Gorbatsevich fibration. Let $M=G / H$, let $H^{0}$ be the identity component of $H$ and let $P(L)=\operatorname{Norm}_{G}\left(L^{0}\right)$ be the normalizer of the identity component of a subgroup $L$ in $G$, with $P^{k}(L)=P\left(P^{k-1}(L)\right)$. We have:

Proposition 4. Let $G$ be a connected real Lie group acting almost effectively and transitively on the manifold $M=G / H$ and let $G / H \rightarrow G / P^{2}(H)$ be the double normalizer fibration. Then

(1) $P^{k}(H)=P^{2}(H)$ for all $k \geq 2$. In particular, the double normalizer fibration of $G / P^{2}(H)$ is a trivial fibering.

(2) $G / P(H)$ is a compact homogeneous space in $\mathbf{R} P^{k}$ for some integer $k$ such that $G$ acts as a linear group and $P(H)$ is a t-subgroup of $G$. In particular, the nilradical of $G$ is in $P(H)$ and the intersection of a semisimple part of $G$ with $P(H)$ is a standard t-subgroup.

(3) Any normalizer bundle of $G / P^{2}(H)$ is itself.

(4) The semisimple part $S$ of $G$ acts on $G / P^{2}(H)$ transitively. There is a maximal connected compact subgroup $K$ of $S$ acting on $G / P^{2}(H)$ transitively, i.e., $G / P^{2}(H)=K / K \cap P^{2}(H)$.

If $G$ is a complex Lie group and $H \subset G$ is a closed complex subgroup, then we have the normalizer fibration $G / H \rightarrow G / P(H)$ and $P^{k}(H)=P(H)$. Let $\mathcal{G}$ and $\mathcal{H}$ denote the Lie algebras of $G$ and $H$, respectively. The base space $G / N$ is realized as the $\operatorname{Ad}(G)$-orbit of the subspace $\mathcal{H}$ in the Grassmann manifold of subspaces of $\mathcal{G}$ that have the same dimension as that of $\mathcal{H}$. Also, $G / P(H)$ is a rational homogeneous manifold and $P(H) / H$ is a compact parallelizable homogeneous manifold.

3.1.4. Foliation with maximal compact subgroup. In this subsection we will recall the foliation fibering induced by the action of the maximal compact subgroup of $G$. 
This was considered by V. V. Gorbatsevich in [8]. He proved following structure theorem:

Proposition 5. Let $M=G / H$ be a compact homogeneous, and let $K$ be a maximal compact Lie subgroup in $G$. Then all the $K$ orbits have same dimension and $M / / K$ is a good orbifold, i.e., it has a compact manifold as a covering. Moreover, there is a subgroup $H^{\prime} \subset H$ of finite index such that all the $K$ orbits on $M^{\prime}=G / H^{\prime}$ are the same and $M^{\prime} / / K$ is smooth with $\mathbf{R}^{k}$ as its universal covering for some integer $k$.

3.1.5. Koszul algebras. In the rest of this paper we will frequently use arguments on the Lie algebra level.

First we recall the following result due to Koszul [21]:

Proposition 6. Let $G$ be a real Lie group and let $H$ be a closed subgroup. Then $G / H$ admits a $G$-invariant symplectic structure if and only if there exist a 2-form $\rho$ on $\mathcal{G}$ which satisfies following conditions for all $x, y, z \in \mathcal{G}$ and $h \in H$ :

$$
\begin{aligned}
& \rho([x, y], z)+\rho([y, z], x)+\rho([z, x], y)=0, \\
& \rho(\operatorname{Ad} h(x), \operatorname{Ad} h(y))=\rho(x, y) .
\end{aligned}
$$

3.1.6. Mal'cev splitting. Here we collect some results we need from the splitting theory of the Lie group (see [9]). Let $G=S R$ be a Levi decomposition of a semisimple Lie group. We call $G$ a splittable Lie group if $R=T U$ with $T \cap U=\{e\}$ such that $T$ acts semisimply and $U$ acts unipotently on the Lie algebra $\mathcal{G}$. We call a Lie group embedding $\alpha: G \rightarrow M(G)$ from $G$ to a splittable simply connected Lie group $M(G)=T \cdot S \cdot U$ a Mal'cev splitting or M-splitting if $\alpha(G)$ is a normal subgroup of $M(G)$ and $M(G)$ is a semidirect product of $T$ and $\alpha(G)$, and $\alpha(G) \cdot U=$ $M(G)$.

Proposition 7. For any simply connected Lie group $G$ there is a unique Mal'cev splitting.

The Mal'cev splitting can be constructed as follows:

Let $G=S \cdot R$ be the Levi decomposition of a connected simply connected Lie group $G$. Consider the adjoint representation $\operatorname{Ad}_{G}: G \rightarrow G L(\mathcal{G})$; put $G^{*}=$ $\operatorname{Ad}_{G}(G)$, and let $\langle G\rangle$ be the algebraic closure of $G^{*}$ in $G L(\mathcal{G})$. Since $\langle G\rangle$ is algebraic, it has a Chevalley decomposition $\langle G\rangle=T^{*} S^{*} U^{*}$, where $U^{*}$ is the unipotent radical, $S^{*}$ is semisimple, and $T^{*}$ is abelian and consists of semisimple (i.e., completely reducible) elements. Put $W^{*}=S^{*} U^{*}$; then $\langle G\rangle=T^{*} W^{*}$, with $T^{*} \cap W^{*}$ finite. Let $t^{*}: T^{*} W^{*} \rightarrow T^{*} / T^{*} \cap W^{*}$ be the natural epimorphism, with kernel $W^{*}$. Writing $\hat{T}=T^{*} / T^{*} \cap W^{*}$, we clearly have $t^{*}(\operatorname{Ad} G) \subset(\hat{T})^{0}$, since $G$ is connected. If for the connected abelian Lie group $\left(T^{*}\right)^{0}$ we consider the universal covering for $\pi_{T}: \tilde{T} \rightarrow$ $\left(T^{*}\right)^{0}$, it is obvious that $t^{*} \cdot \pi_{T}: \tilde{T} \rightarrow(\hat{T})^{0}$ is the universal covering for $(\hat{T})^{0}$. Since $G$ is connected and simply connected, there exists a unique homomorphism $\tilde{t}: G \rightarrow \tilde{T}$ such that $t^{*} \cdot \pi_{T} \cdot \tilde{t}=t^{*} \cdot \operatorname{Ad}_{G}$. Put $T=\tilde{t}(G), T_{G}^{*}=\pi_{T} \cdot \tilde{t}(G)$; then $T$ is a connected simply connected abelian Lie group covering of $T^{*}$, while $T_{G}^{*} \subset\langle G\rangle$. We see that $T_{G}^{*}$ can be regarded as a subgroup of Aut $G$. The imbedding $T_{G}^{*} \rightarrow \operatorname{Aut} G$ and the homomorphism $\pi_{T}$ induce a homomorphism $\phi: T \rightarrow \operatorname{Aut} G$, with $\operatorname{ker} \phi=\operatorname{ker} \pi_{T} \cap T$ discrete. Then we can get the Mal'cev splitting $M(G)=T \times_{\phi} G$ and $M(G)=T S U$ for a unipotent group $U$ such that $\operatorname{dim} U=\operatorname{dim} G, \operatorname{dim} U / N_{R}=\operatorname{dim} T$. Now we let $W_{G}=S U, W_{G, l}=(S / l(S)) \cdot U$, where $l(S)$ is in the center of $S$ such that 
$S_{l}=S / l(S)$ is linear. Then Aut $W_{G, l}$ and the semidirect product Aut $W_{G, l} \propto W_{G, l}$ are prealgebraic groups, i.e., the identity components of algebraic groups. We can regard $T_{G}^{*}$ as a subgroup of Aut $W_{G, l}$. Let $a\left(T_{G}^{*}\right)$ be the prealgebraic hull of $T_{G}^{*}$ in Aut $W_{G, l}$, and $\mathcal{A}_{l}(G)=a\left(T_{G}^{*}\right) \propto W_{G, l}$. We see that $\mathcal{A}_{l}(G)$ is prealgebraic. Let $M_{l}(G)=T_{G}^{*} S_{l} U$ as a quotient of $M(G)$. Then:

Proposition 8. The group $\mathcal{A}_{l}(G)$ is prealgebraic, and there exists an imbedding $\beta: M_{l}(G) \rightarrow \mathcal{A}_{l}(G)$ such that the following properties hold:

(1) $\mathcal{A}_{l}(G)$ is splittable, and if $\mathcal{A}_{l}(G)=T^{\prime} S^{\prime} U^{\prime}$, where $U^{\prime}$ is unipotent, $S^{\prime}$ is semisimple and $T^{\prime}$ is a prealgebraic torus, then $\beta\left(M_{l}(G)\right) \supset S^{\prime} U^{\prime}$ and $S^{\prime}=$ $S_{l}$, where $S$ is the semisimple part of $G$ and $U^{\prime}=U$.

(2) The prealgebraic closure of each of the subgroup $\beta\left(G_{l}\right)$ and $\beta\left(M_{l}(G)\right)$ in $\mathcal{A}_{l}(G)$ is $\mathcal{A}_{l}(G)$ itself.

Here we would like to give a very simple example: Let $G=G_{1} \times G_{2}, G_{1}=T N$ with $T, N, G_{2}$ abelian and $T$ acts on $N$ almost faithfully and as a compact torus without any eigenvector. Then $\langle G\rangle=\operatorname{Ad}_{G}(T) N, W^{*}=N$,

$$
\begin{gathered}
t^{*}: \operatorname{Ad}_{G}(T) N \rightarrow \operatorname{Ad}_{G}(T)=\hat{T}, \\
\pi_{T}: T \rightarrow \operatorname{Ad}_{G}(T), \\
\tilde{t}: T N \times G_{2} \rightarrow T, \\
T_{G}^{*}=\operatorname{Ad}_{G}(T), \phi: T \rightarrow \operatorname{Ad}_{G}(T), \\
M(G)=T \times_{\phi} G=T U, U=\left\{\left.\left(t, t^{-1}, n, g\right)\right|_{t \in T} n \in N g \in G_{2}\right\}, \\
W_{G}=W_{G, l}=U, \mathcal{A}_{l}(G)=M_{l}(G)=\operatorname{Ad}_{G}(T) U .
\end{gathered}
$$

3.1.7. Modifications. The Gorbatsevich modification for a compact homogeneous space was first used in [10. Similar construction can be found in the study of homogeneous Kähler manifolds, e.g., [5], [6].

Let $M=G / H$ be a compact homogeneous space of a simply connected Lie group $G$. We let $G_{*}=G_{l}=G / l(S)$ be the image of $G\left(H_{*}=H / H \cap l(S)\right.$ be the image of $H)$ in $\mathcal{A}_{l}(G)$. We also let $P_{*}=N_{\mathcal{A}_{l}(G)}\left(H_{*}^{0}\right)$, the normalizer of $H_{*}^{0}$. Since the subgroup $H_{*}^{0}$ is connected, then its normalizer is a prealgebraic group, i.e., the identity component of an algebraic group. Hence the group $\pi_{0}\left(P_{*}\right)$ is finite. Passing from $H$ to the subgroup $H_{1}=H \cap \pi^{-1}\left(P_{*}^{0} \cap H_{*}\right)$ of finite index, where $\pi: M(G) \rightarrow M_{l}(G)$ is the natural epimorphism, we might assume that $H_{*} \subset P_{*}^{0}$ by considering a finite covering $M^{\prime}$ of $M$. This inclusion will be assumed to hold in what follows.

Consider the natural epimorphism $\gamma: \mathcal{A}_{l}(G) \rightarrow \mathcal{A}_{l}(G) / W_{l}$. We have

$$
\mathcal{A}_{l}(G) / W_{l}=T_{*} \times \pi\left(W_{G}\right) / W_{l}
$$

with $W_{G}=S U, W_{l}=S_{l} N_{R}$ (our $W_{l}$ is the same as in [10] but different from the one in [9] where $W_{l}=S / l(S) \cdot U$ ) and $T_{*}$ is a prealgebraic torus; $\pi\left(W_{G}\right) / W_{l}=U / N_{R}$. So $\operatorname{Im} \gamma=T_{*} \times U / N_{R}$; we denote it by $A$. $A$ is connected and Abelian. There is a natural embedding of the group $G_{*} / W_{l}=R / N_{R}$ in $M_{l}(G) / W_{l}$ which is contained in $A$.

Denote the image of $R / N_{R}$ by $B$. By $B \cap T_{*}=\{e\}$ we see that the projection $\mu: T_{*} \times U / N_{R} \rightarrow U / N_{R}$ to the second factor is an isomorphism on $B$, i.e., $B$ is closed in $A$. Now consider the subgroup $H_{*} / H_{*} \cap W_{l}$ of $A$ and its closure $\overline{H_{*} / H_{*} \cap W_{l}}$ (in the Euclidean topology) which we denote by $A_{1}$. Since $H_{*} / H_{*} \cap W_{l} \subset B$ we 
have $A_{1} \subset B$. Since the group $B$ is simply connected and Abelian, $A_{1}$ is a closed subgroup of it, and $A_{1}$ is torsion free and isomorphic to $\mathbf{R}^{p} \times \mathbf{Z}^{q}$ for some $p, q \geq 0$.

Finally we consider the subgroup $\gamma\left(P_{*}\right) \subset A$. The subgroup Ker $\gamma=W_{l}$ is closed in the "Zariski topology" on $\mathcal{A}_{l}(G)$; so is the Lie group $P_{*}$, therefore $\gamma\left(P_{*}\right)$ is a closed subgroup of $A$. But $H_{*} \subset P_{*}$, so $H_{*} / H_{*} \cap W_{l} \subset \gamma\left(P_{*}\right)$ and hence $A_{1} \subset \gamma\left(P_{*}\right)$, i.e., $A_{1} \subset \gamma\left(P_{*}^{0}\right)$ by our convention. The group $\gamma\left(P_{*}^{0}\right)$ is connected and Abelian and hence $\gamma\left(P_{*}^{0}\right)=K \times V$, where $K$ is a maximal compact subgroup of $\gamma\left(P_{*}^{0}\right)$ (which is a torus), and $V$ is simply connected. Since $A_{1}$ is closed in $A$ and torsion free, $A_{1} \cap K=\{e\}$. Hence the projection $K \times V \rightarrow V$ onto the second direct factor on $A_{1}$ is a monomorphism. Now it follows from this that there exists a closed simply connected subgroup $C \subset \gamma\left(P_{*}^{0}\right)$, such that $A_{1} \subset C$ and $A_{1}$ is uniform in $C$ (we notice that $C$ is not always in $B$ ). We set $\Phi_{l}=\gamma^{-1}(C)$. Then $\Phi_{l}$ is a closed connected subgroup of $\mathcal{A}_{l}(G)$. To it corresponds a closed connected subgroup $\Phi$ of $\mathcal{A}(G)$.

With this construction at hand, V. V. Gorbatsevich proved in [10] the following theorem:

Proposition 9. Let $M=G / H$ be a compact homogeneous space of a simply connected Lie group $G$. Then there exists a subgroup $H^{\prime}$ of finite index in $H$ and a subgroup $\Phi$ of $\mathcal{A}(G)$, such that:

(1) $\Phi$ is a connected, simply connected, closed subgroup of $\mathcal{A}(G)$, containing $H^{\prime}$,

(2) $W_{\Phi}=W_{G}$, in particular $S_{\Phi}=S_{G}, U_{\Phi}=U_{G}($ although $M(\Phi)$ and $M(G)$ are not generally isomorphic),

(3) for the decomposition $\mathcal{A}(G)=T W_{G}$ with $T$ an Abelian subgroup of $\mathcal{A}(G)$, we have $\Phi \subset T G, G \subset T \Phi$, where $\Phi \cap T=G \cap T=\{e\}$,

(4) there exists a diffeomorphism $\eta: \Phi \rightarrow G$ which is the identity on the subgroup $H^{\prime}$ and induces a diffeomorphism $\Phi / H^{\prime} \rightarrow G / H^{\prime}$,

(5) $\Phi=N_{\Phi}\left(\left(H^{\prime}\right)^{0}\right) S_{\Phi}$,

(6) the Lie group $\left(N_{\Phi}\left(\left(H^{\prime}\right)^{0}\right)\right)_{l}=N_{\Phi}\left(\left(H^{\prime}\right)^{0}\right) / N_{\Phi}\left(\left(H^{\prime}\right)^{0}\right) \cap l(S)$ has a finite number of connected components.

Here we also test this construction with a simple example that $G$ is the same as the example in last subsection and $H$ is in the kernel of $\left.\operatorname{Ad}\right|_{\mathcal{N}}$ such that $\mathcal{H} \subset \mathcal{N}$ and does not contain any ideal of $\mathcal{G}_{1}, N_{\mathrm{Ad}(T)}\left(H_{*}^{0}\right)$ is discrete. Then:

$$
\begin{gathered}
G_{*}=G_{l}=\left\{\left.\left(\operatorname{Ad} t,\left(t, t^{-1}\right), n, g\right)\right|_{t \in T} n \in N g \in G_{2}\right\}, \\
H_{*}=H, H_{*} \subset\left\{\left.\left(\operatorname{Ad} t,\left(t, t^{-1}\right), n, g\right)\right|_{\operatorname{Ad} t=1}\right\} \subset U, \\
P_{*}=N_{\operatorname{Ad}(T)}\left(H_{*}^{0}\right) U, P_{*}^{0}=U, H_{1}=H, M^{\prime}=M, W_{G}=U, W_{l}=N, \\
\gamma: \operatorname{Ad}(T) U \rightarrow \operatorname{Ad}(T) \times U / N=A=\left\{\left.\left(\operatorname{Ad} t_{1},\left(t_{2}, t_{2}^{-1}\right), g\right)\right|_{t_{1}, t_{2} \in T} g \in G_{2}\right\}, \\
T_{*}=\operatorname{Ad}(T), G_{*} / N_{\mathbf{R}}=\left\{\left.\left(\operatorname{Ad} t,\left(t, t^{-1}\right), g\right)\right|_{t \in T} g \in G_{2}\right\}=B, \\
A_{1}=\overline{H_{*} / H_{*} \cap W_{l}}=H_{*} / H_{*} \cap W_{l} \subset\left\{\left.\left(\operatorname{Ad} t,\left(t, t^{-1}\right), g\right)\right|_{\mathrm{Ad} t=1}\right\}, \\
p=0, K=e, V=U / N, C=V . \Phi_{l}=U=\Phi .
\end{gathered}
$$

This modification turns out to be very useful in our classification.

3.2. Theorem 1. Let $(G / H, \omega)$ be a compact homogeneous space with an invariant symplectic structure. Then the double normalizer bundle is a product of a rational homogeneous space and a solv-manifold with an invariant symplectic structure. 
Proof. Assume that $G$ is simply connected. Then the maximal compact subgroup $K$ is semisimple. Therefore $K$ is Poisson. By Proposition 5 and (4) of section 2.1 we get that every $K$ orbit is, up to a finite covering, a same torus bundle over a rational homogeneous space $Q=K / J$ which is induced by the moment map $K / K \cap H \rightarrow K / J$. In particular, if $\xi \in \mathcal{J}$ (the Lie algebra of $J$ ) and if $T$ is the closure of the 1-parameter group $\exp t \xi$ in $J$, then

$$
\operatorname{Fix}_{M}(T)=\left\{x \mid \xi_{M}(x)=0\right\}=\left\{x \mid d f_{\xi}(x)=0\right\} \neq 0 .
$$

Now let $T$ be a maximal torus in $J$. We see that one of the $K$ orbit must be $Q$ since no other homogeneous space of this type can be locally isomorphic to $Q$ (the isotropy group $J$ is connected and is the normalizer of itself). So all the $K$ orbits are isomorphic to $Q$. For any $K$ orbit there is only one point on it with isotropy group $J$. So we get a section $s$ of the compact foliation, i.e., $M$ is a product of $Q$ and $s$. Now we see that $s$ is a symplectic reduction of the moment map and therefore $s$ is a symplectic manifold with the induced symplectic structure.

From the normalizer of $J$ being itself, i.e., $J=K \cap H=K \cap P^{2}(H)$, we see that the double normalizer fibration (see (4) of Proposition 4) has $Q$ as the base and $s$ as a fiber and $s$ itself is a compact homogeneous space with an invariant symplectic structure. We have $s=P^{2}(H) / H$ as a fiber of the double normalizer fibration. By $P(H)$ being a t-subgroup in $G$ we see that all the semisimple factors of $P^{2}(H)$ are in $P(H)$. If a simple factor $S_{1}$ of $P(H)$ is not in $H$ it acts almost freely on $s$, i.e., only elements in its center may have fixed points. Then since $S_{1}$ is Poisson, every element in its Lie algebra has fixed points, a contradiction. We see that all the semisimple factors must be in $H$, i.e., the radical of $P^{2}(H)$ acts on $s$ transitively. So we get that $s$ is a solv-manifold with an invariant symplectic structure.

Remark 1. (1) In our proof above we had to use the structure of the double normalizer fibration and t-subalgbra in Propositions 3 and 4 to prove that the moment map is actually equivariant.

(2) An easy calculation can show that the Levi decomposition is actually a product. We first proved this in [17], and later found that this is a conclusion of our Theorem. See also [24].

3.3. Compact solv-manifolds with invariant symplectic structures. Here we shall deal with the second factor in Theorem 1.

Lemma 2. Every compact solv-manifold with an invariant symplectic structure is a two-step solv-manifold. Moreover, the orbits of the commutator are isotropic, and any element in the Lie algebra corresponding to the commutator is conjugate to an element in the Lie algebra of the isotropy group.

Proof. We consider the moment map introduced by $\mathcal{N}=[\mathcal{G}, \mathcal{G}]$ (see the sentence after (3) of section 2.1). The corresponding subgroup $N$ is unipotent, in particular on $\mathcal{N}^{*}$, and each orbit in the image is compact, hence must be a point. We see that $N / N \cap H$ is abelian, $[\mathcal{N}, \mathcal{N}] \subset \mathcal{H}$ as an ideal must be zero. Hence $G$ is a two-step solvable group. Moreover, since the moment map is a constant on each commutator orbit, we see that $d f(X)=\omega\left(X_{f}, X\right)=0$ for all $X_{f}, X$ in the commutator, i.e., the commutator orbits are isotropic.

We also see that any element in the Lie algebra of the commutator corresponds to a function on the manifold, and hence has a zero point, i.e., this element is in the Lie algebra of the isotropy group at that point. So every element in the Lie algebra 
of the commutator is conjugate to an element in the Lie algebra of the isotropy group.

Now we assume that $\mathcal{G}$ is a direct $\operatorname{sum} \mathcal{A}+\mathcal{N}$ as a vector space, where $\mathcal{N}=[\mathcal{G}, \mathcal{G}]$ and such that $\mathcal{H}=\mathcal{H} \cap \mathcal{A}+\mathcal{H} \cap \mathcal{N}$. We let $\mathcal{H}_{1}=\mathcal{H} \cap \mathcal{A}, \mathcal{H}_{2}=\mathcal{H} \cap \mathcal{N}$ and

$$
\mathcal{B}=\left\{\left.a \in \mathcal{A}\right|_{\rho(a, \mathcal{N})=0}\right\},
$$

$\mathcal{B}=\mathcal{H}_{1}+\mathcal{A}_{1}$ as a direct sum of vector spaces. Then $\mathcal{A}$ is a direct sum $\mathcal{H}_{1}+\mathcal{A}_{1}+\mathcal{A}_{2}$, $\mathcal{N}$ is a direct sum $\mathcal{H}_{2}+\mathcal{N}_{1}$ as vector spaces, where $\mathcal{A}_{2}$ is a complement of $\mathcal{H}_{1}$ in

$$
\mathcal{C}=\left\{\left.a \in \mathcal{A}\right|_{\rho\left(a, \mathcal{A}_{1}\right)=0}\right\}
$$

We have:

Lemma 3. $[\mathcal{B}, \mathcal{B}]=0,[\mathcal{B}, \mathcal{N}]=0$.

Proof. Since $\rho([\mathcal{B}, \mathcal{B}], \mathcal{A})=\rho(\mathcal{B},[\mathcal{B}, \mathcal{A}]) \subset \rho(\mathcal{B}, \mathcal{N})=0$, we see that $[\mathcal{B}, \mathcal{B}] \subset \mathcal{H}$. In the same way we see that $[\mathcal{B}, \mathcal{N}] \subset \mathcal{H}$. Also, $[[\mathcal{B}, \mathcal{B}], \mathcal{G}] \subset[\mathcal{B}, \mathcal{N}],[[\mathcal{B}, \mathcal{N}], \mathcal{G}] \subset$ $[\mathcal{B}, \mathcal{N}]$. We see that $[\mathcal{B}, \mathcal{N}],[\mathcal{B}, \mathcal{B}]$ generates an ideal in $\mathcal{H}$, that is, $[\mathcal{B}, \mathcal{B}]=$ $[\mathcal{B}, \mathcal{N}]=0$

From Lemma 3 it follows that $\mathcal{H}_{1}$ is a Lie subalgebra; by modification we may assume that $\mathcal{H}_{1}=0$. We also see that $\mathcal{A}_{1} \subset P(\mathcal{H})$. By the last sentence of Lemma 1 and counting the dimension, $P(\mathcal{H})=\mathcal{N}+\mathcal{A}_{1}$. Consider the $G$ action on the Grassmanian $G(\mathcal{G}, \mathcal{H})$ of $\mathcal{H}$ in $\mathcal{G}$. The orbit through $\mathcal{H}$ is exactly the base of the normalizer fibering. Being compact and with an abelian transitive group, it must be a torus with its action on itself. We can also regard it as an orbit in $G(\mathcal{N}, \mathcal{H})$, and the action of $G$ on it is an almost faithful representation of adjoint action $\left.\operatorname{Ad} G\right|_{\mathcal{N}}$ on $\mathcal{N}$ as the restriction of the adjoint action. In particular, we see that Ad $G$ acts on $\mathcal{N}$ as a compact group. To see this we notice that $H\left(N+A_{1}\right)$ is an open and closed subgroup of $P(\mathcal{H})$, hence is closed in $G$, i.e., $G / H\left(N+A_{1}\right)$ is compact. We see that $H\left(N+A_{1}\right)$ is cofinite in $P(\mathcal{H})$. But $H$ acts on $\mathcal{N} / \mathcal{H}$ trivially since $\left(\mathcal{A}_{2}, \mathcal{N} / \mathcal{H}\right)$ is a perfect pair to $\rho$ and any invariant subspace of $\mathcal{H}$ on which $H$ acts nontrivially must be an ideal of $\mathcal{G}$, i.e., $H$ is in the kernel of $\left.\operatorname{Ad}\right|_{\mathcal{N}}$. Now for a generic element in $G$, it does not have any eigenvector in $\mathcal{N}$, otherwise this eigenvector will generate an ideal in $\mathcal{H}$. Now we consider a minimal invariant subspace $V$ of the linear transformation of $A=\operatorname{ad}(a)$ on $\mathcal{G}$ for an element $a \in \mathcal{A}_{2}$; then either $\left.\operatorname{ad}(a)\right|_{V}=0$ or $V \cap \mathcal{N} \neq 0$. In the latter case we must have $V \subset \mathcal{N}$ since the eigenvalues of $\left.\operatorname{ad}(a)\right|_{V \mathbf{C}}$ are not zero, hence $V$ has dimension 2 and $\operatorname{ad}(a)$ is semisimple. This implies that $\mathcal{A}_{1}$ can be chosen to be an abelian ideal, and $\mathcal{A}_{2}+\mathcal{N}$ is another ideal. So we get:

Lemma 4. If we let $\mathcal{G}_{1}=\mathcal{A}_{2}+\mathcal{N}$ and $\mathcal{G}_{2}=\mathcal{A}_{1}$, then $\mathcal{G}=\mathcal{G}_{1}+\mathcal{G}_{2}$ as direct sum of Lie algebras. Moreover, $\mathcal{G}_{2}$ is abelian and $\mathcal{A}_{2}$ acts on $\mathcal{G}_{1}$ as torus.

We will apply the Gorbatsevich modification (Proposition 9) to our case with some modification from section 3.1.7. Instead of $P_{*}=N_{\mathcal{A}_{l}(G)}\left(H_{*}^{0}\right)$ we consider $P_{*} \cap D$, where

$$
D=\left\{\left.a \in \mathcal{A}_{l}(G)\right|_{\rho(\operatorname{Ad}(a) x, \operatorname{Ad}(a) y)=\rho(x, y) \text { for all } x, y \in \mathcal{G}\}} .\right.
$$

Then all the construction will go through, and basically we get same modification modulo a finite covering. In the case of the example in section 3.1.7 we see in particular that $P_{*}^{0}=U$ acts trivially on $N$. Later on we will see that this is what exactly happens here. 
Moreover if we defined $\rho\left(t_{1}+x, t_{2}+y\right)=\rho(x, y)$ on $M(G)$, then on $\Phi$ we have $\rho\left(\left[t_{1}+x, y\right], z\right)+\rho\left(y,\left[t_{1}+x, z\right]\right)=0$ for all $t_{1}+x \in \operatorname{Lie} \Phi, y, z \in \mathcal{G}$ and hence

$$
\begin{aligned}
\rho\left(\left[t_{1}\right.\right. & \left.\left.+x, t_{2}+y\right], t_{3}+z\right)+\rho\left(\left[t_{2}+y, t_{3}+z\right], t_{1}+x\right) \\
& +\rho\left(\left[t_{3}+z, t_{1}+x\right], t_{2}+y\right) \\
& =\rho\left(\left[t_{1}+x, t_{2}+y\right], z\right)+\rho\left(\left[t_{2}+y, t_{3}+z\right], x\right) \\
& +\rho\left(\left[t_{3}+z, t_{1}+x\right], y\right) \\
& =\rho\left(\left[t_{1}+x, y\right], z\right)+\rho\left(\left[x, t_{2}+y\right], z\right)-\rho([x, y], z) \\
& +\rho\left(\left[t_{2}+y, z\right], x\right)+\rho\left(\left[y, t_{3}+z\right], x\right)-\rho([y, z], x) \\
& +\rho\left(\left[t_{3}+z, x\right], y\right)+\rho\left(\left[z, t_{1}+x\right], y\right)-\rho([z, x], y) \\
& =0
\end{aligned}
$$

for all $t_{1}+x, t_{2}+y, t_{3}+z \in \operatorname{Lie} \Phi$.

Hence, $H$ is in the kernel of $\left.\mathrm{Ad}\right|_{\mathcal{N}}$. From the example in section 3.1.7 we have that in our case $\Phi$ is abelian and $\rho\left(\operatorname{Ad}(h)\left(t_{1}+x\right), \operatorname{Ad}(h)\left(t_{2}+y\right)\right)=\rho\left(t_{1}+x, t_{2}+y\right)$. Applying Proposition 7 we see that $\Phi / H$ is also a compact homogeneous space with an invariant symplectic structure. Since $\Phi$ is abelian it is a torus. So we get:

Theorem 2. Every solvable compact homogeneous space with an invariant symplectic structure is a torus.

Remark 2. The actual symplectic torus structure was obtained in 17 by a completely integrable system. See also 24] for a very interesting version of the proof of Theorem 2.

Combining Theorems 1 and 2 we obtain Proposition 2 .

3.4. Examples of nonstandard symplectic torus. In last section we obtained nonstandard symplectic tori in the case $\mathcal{A}_{2} \neq 0$ and $\mathcal{H}_{2} \neq 0$. Although they have standard tori as their modification, they themselves are not standard. The dimension of $\mathcal{H}_{1}$ can be as big as possible, and so can the dimension of $G$. In this section we will give some simple examples which show that this situation does occur.

Let $\mathbf{C}^{n}$ be a complex vector space generated by vectors $e_{1}, \cdots, e_{n}$ with $S_{1}=$ $\left\{\left.e^{i \theta}\right|_{\theta \in[0,2 \pi]}\right\}$ action $e^{i \theta}: e_{k} \rightarrow e^{k i \theta} e_{k}$.

Regarding $S_{1}$ as a subgroup of the automorphism group of the abelian group $\mathbf{C}^{n}$ we get a Lie group $G=S_{1} \propto \mathbf{C}^{n}$ as a semiproduct.

Let $Z=(\mathbf{Z}+i \mathbf{Z})^{n}$ be the standard lattice in $\mathbf{C}^{n}$,

$$
H^{0}=\left\{\left.\left(z_{1}, \cdots, z_{n}\right) \in \mathbf{C}^{n}\right|_{\operatorname{Re}\left(z_{1}+\cdots+z_{n}\right)=0}\right\},
$$

and $H=Z+H^{0}$.

Then $G / H$ is a nonstandard torus which occured in the last section, with a symplectic structure introduced by $\rho$ in Proposition 7 , such that

$$
\rho(s, x)=1, \rho(s, h)=0, \rho(x, h)=0
$$

for all $h \in \mathcal{H}$, where $s$ is a generator of the Lie algebra of $S_{1}$, and $x \in \mathbf{C}^{n}$ is an element such that $\operatorname{Re}\left(x_{1}+\cdots+x_{n}\right)=1$.

See also [24] for similar examples.

It is a very surprising fact to me that we can get a classification for compact homogeneous space with an invariant symplectic structure since the automorphism group of a compact symplectic manifold has infinite dimension. From any smooth 
function we can construct a one-parameter group which keeps the symplectic structure invariant. So we can see that the base manifold is always "homogeneous" under the symplectic automorphism group. But we see from our classification that a compact symplectic manifold that is homogeneous under a finite-dimensional Lie subgroup of the symplectic automorphism group is so different. Moreover, we seem to be able to classify a compact homogeneous space with a symplectic structure which is not necessarily invariant under the group action.

Using the nonstandard torus we can construct new examples of simply-connected compact symplectic manifolds as in [15] and [1].

\section{ACKNOWLEDGEMENT}

Here I like to take this opportunity to thank Professor A. T. Huckleberry for giving a simpler proof of the main result in 3] and Professor J. Dorfmeister for leading me into the area of homogeneous spaces; those efforts made this paper possible. I express my thanks to the Department of Mathematics, Princeton University, Professor W. C. Hsiang, the Department of Mathematics, University of California at Riverside, and to Professor B. Wong for their support. I also thank Professors Bogomolov, Ding, Gromov, Jiang, Kobayashi, Siu and Wolf for their constant support. I also thank the referees for their helpful comments and Professor A. R. Stralka for helping with the language in both this paper and [18].

\section{REFERENCES}

1. F. A. Bogomolov: On Guan's Examples of Simply connected Non-Kähler Compact Complex Manifolds, Amer. J. Math. 118 (1996), 1037-1046. MR1408498|(97k:32048)

2. A. Borel \& R. Remmert: Über Kompakte Homogene Kählersche Mannigfaltigkeiten, Math. Ann. 145 (1962), 429-439. MR0145557 (26:3088)

3. J. Dorfmeister \& Z. Guan: Classifications of Compact Homogeneous Pseudo-Kähler Manifolds, Comm. Math. Helv. 67 (1992), 499-513. MF1185806 (93i:32042)

4. J. Dorfmeister \& Z. Guan: Pseudo-Kählerian Homogeneous Spaces Admitting a Reductive Transitive Group of Automorphisms, Math. Zeischrift 209 (1992), 89-100. MR1143216 (92k:32058)

5. J. Dorfmeister: Homogeneous Kähler Manifolds Admitting a Transitive Solvable Group of Automorphisms, Ann. Scient. Ec. Norm. Sup., 4 Serie, vol. 18 (1985), 143-180. MR0803198 (87j:32094)

6. J. Dorfmeister \& K. Nakajima: The Fundamental Conjecture for Homogeneous Kähler Manifolds, Acta Math. 161 (1988), 23-70. MR0962095 (89i:32066)

7. V. V. Gorbatsevich: On the Double Normalizer of the Stationary Subalgebra of a Plesiocompact Homogeneous Spaces, Siberian Math. J. 34 (1993), 451-456. MR 1241168 (94h:53063)

8. V. V. Gorbatsevich: On a Fibration of Compact Homogeneous Spaces, Trans. Moscow Math. Soc. vol. 1 (1983), 129-157.

9. V. V. Gorbatsevich: Splittings of Lie Groups and Their Application to the Study of Homogeneous Spaces, Math. USSR Izvestija. vol. 15 (1980), 441-467.

10. V. V. Gorbatsevich: Plesiocompact Homogeneous Spaces, Siber. Math. J. 30 (1989), 217-226. MR0997468 (90f:22010)

11. V. Guillemin \& S. Sternberg: Symplectic Techniques in Physics, Cambridge Univ. Press. 1984. MR0770935 (86f:58054)

12. Z. Guan: Examples of compact holomorphic symplectic manifolds which admit no Kähler structure. In Geometry and Analysis on Complex Manifolds-Festschrift for Professor S. Kobayashi's 60th Birthday, World Scientific, 1994, 63-74. MR1463964 (98h:53109)

13. D. Guan: A Splitting Theorem for Compact Complex Homogeneous Spaces with a Symplectic Structure. Geom. Dedi. 67 (1996), 217-225. MR1413633 (98a:53105)

14. D. Guan: Classification of Compact Complex Homogeneous Spaces with Invariant Volumes, Transactions of AMS. 254 (2002), 4493-4504. MR.1926885 (2003h:32035) 
15. D. Guan: Examples of Compact holomorphic Symplectic Manifolds which are not Kählerian II, Invent. Math. 121 (1995), 135-145. MR1345287 (97i:32033)

16. D. Guan: Classification of Compact Homogeneous Space with an Invariant Symplectic Structure, preprint 1997, An Announcement appeared in ERA-AMS vol. 3 (1997), 52-54. MR 1464575 (99a:53065)

17. D. Guan: Fine Structure of Compact Homogeneous Space with an Invariant Symplectic Structure, preprint 1997.

18. D. Guan: Toward a Classification of Compact Complex Homogeneous Spaces, J. Algebra vol. 273 (2004), 33-59. MR2032450

19. A. T. Huckleberry: Homogeneous Pseudo-Kählerian Manifolds: A Hamiltonian Viewpoint, Note di Matematica 10(1990) suppl. 2, 337-342. MR1221949 (94f:53052)

20. A. T. Huckleberry \& E. Oeljeklaus: Classification Theorems for Almost Homogeneous Spaces, Publ. de l'Inst. Elie Cartan, Nancy, Janvier 1984, 9. 178 pages. MR.0782881 (86g:32050)

21. J. L. Koszul: Sur la Form Hermitienne Canonique des Spaces Homogenes Complexes, Canad. J. Math. 7 (1968), 562-576. MR0077879(17:1109a)

22. A. L. Onishchik: On Lie Groups Transitive on Compact Manifolds II, Math. USSR Sbornik. vol. 3 (1967), 373-388.

23. J. Tits: Espaces Homogènes Complexes Compacts, Comm. Math. Helv. 37 (1962), 111-120. MR0154299 (27:4248)

24. Ph. B. Zwart \& W. M. Boothby: On Compact, Homogeneous Symplectic Manifolds, Ann. Inst. Fourier Grenoble 30, 1 (1980), 129-157. MR0576076 (81g:53040)

Department of Mathematics, University of California-Riverside, Riverside, CaliforNIA 92521

E-mail address: zguan@math.ucr.edu 